\title{
Sfil genomic cleavage map of Escherichia coli K-12 strain MG1655
}

\author{
Jeffrey D.Perkins, Joe Don Heath, Baldev R.Sharma and George M.Weinstock* \\ Department of Biochemistry and Molecular Biology, University of Texas Medical School, University of \\ Texas Health Science Center at Houston, 6431 Fannin Street, PO Box 20708, Houston, TX 77225, \\ USA
}

Received October 14, 1991; Revised and Accepted January 29, 1992

\begin{abstract}
An Sfil restriction map of Escherichia coli K-12 strain MG1655 is presented. The map contains thirty-one cleavage sites separating fragments ranging in size from $407 \mathrm{~kb}$ to $3.7 \mathrm{~kb}$. Several techniques were used in the construction of this map, including CHEF pulsed field gel electrophoresis; physical analysis of a set of twenty-six auxotrophic transposon insertions; correlation with the restriction map of Kohara and coworkers using the commercially available $E$. coli Gene Mapping Membranes; analysis of publicly available sequence information; and correlation of the above data with the combined genetic and physical map developed by Rudd, et al. The combination of these techniques has yielded a map in which all but one site can be localized within a range of $\pm 2 \mathrm{~kb}$, and over half the sites can be localized precisely by sequence data. Two sites present in the EcoSeq5 sequence database are not cleaved in MG1655 and four sites are noted to be sensitive to methylation by the $\mathrm{dcm}$ methylase. This map, combined with the Notl physical map of MG1655, can aid in the rapid, precise mapping of several different types of genetic alterations, including transposon mediated mutations and other insertions, inversions, deletions and duplications.
\end{abstract}

\section{INTRODUCTION}

A genomic physical map can aid genetic as well as physical analysis by providing a rapid, precise method of localizing single gene mutations or larger-scale rearrangements of DNA, such as insertions, deletions, inversions or duplications. In organisms that lack a well-developed genetic system, physical mapping techniques may offer one of the few ways of studying the organism's genetic material and detecting and localizing changes caused by various mutational events. In Escherichia coli, a very detailed genetic map, containing over 1,400 genetic loci (1), has evolved along with the development of techniques for manipulating the $E$. coli genome. Nevertheless, the utility of welldeveloped genetic techniques and a detailed genetic map can be enhanced by physical mapping. For example, the mapping of mutations can often be accomplished in less time with physical mapping techniques than with classical genetic approaches. With over one third of the $E$. coli chromosome sequenced to date (K. Rudd, personal communication), and with work underway to sequence the entire $E$. coli genome, the correlation between the genetic and physical maps becomes increasingly firm.

Several tools have been developed over the last few years that facilitate construction of a restriction map of $E$. coli. These mapping tools originated with the restriction map of $E$. coli $\mathrm{K}-12$ strain W3110 constructed by Kohara, et al. using eight frequently cutting enzymes $(2,3)$. Closely connected with this map, Rudd and coworkers have developed a consolidated $E$. coli sequence database and a combined physical and genetic map of $E$. coli based on the Kohara map $(4,5)$. Finally, a membrane containing the miniset of the ordered phage $\lambda$ library of Kohara, et al. has recently become available commercially (6).

One problem inherent in making a physical map of $E$. coli is the existence of thousands of laboratory strains of $E$. coli $\mathrm{K}-12$ that differ from each other in having several mutations and chromosomal rearrangements $(7,8)$. Among currently available strains, EMG2, a strain used by Clowes and Hayes in the 1960's in their microbial genetics course, is commonly considered to be the closest to the original wild type K-12 isolated in 1922 (9; B. Bachmann, personal communication). Strain EMG2 is both $\lambda^{+}$and $\mathrm{F}^{+}$, as was the original $\mathrm{K}-12$ isolate. Strain W1485, derived by Lederberg from his wild type K-12 strain WG1, has been cured of the $\lambda$ prophage, but is still $\mathrm{F}^{+}(8)$. Neither EMG2 nor W1485 is used commonly for genetic experimentation. Strain MG1655 (10), derived directly from W1485, is both $\lambda^{-}$and $\mathrm{F}^{-}$, but is otherwise believed to be representative of wild type $E$. coli $\mathrm{K}-12$. It is widely used as a wild type $E$. coli in genetic studies (e.g., 11, 12). Because of these considerations, we have concentrated our physical mapping efforts on this strain.

This work presents the third complete restriction enzyme cleavage map of MG1655 to be published. The AvrII map was constructed by Daniels (13). The NotI map was constructed by Heath, et al. (14). Both the NotI map and the SfiI map have been correlated with the combined genetic and physical map of Rudd,

* To whom correspondence should be addressed 
et al. $(4,5)$. The mapping of multiple restriction sites in MG1655 will further the physical analysis of MG1655 and its immediate derivatives. It is also anticipated that, by comparison with the MG1655 maps, the analysis of the similarities, differences, and relationships among many common laboratory strains will be facilitated. This analysis will, in turn, yield a detailed and comprehensive picture of these strains in relation to one another.

\section{MATERIALS AND METHODS}

\section{Bacterial strains and media}

Strain GM271 (E. coli genetic stock center number 6477) was obtained from B. Bachmann. The GM271 genotype is: ara-14, leuB6, fhuA31, lacY1, tsx-78, supE44, galK2, galT22, dcm-6, hisG4, rfbD1, rpsL136, xyl-5, mtl-1, thi-1, hsdR2. All other strains used in this study were derived from Escherichia coli K-12 strain MG1655, provided by K. B. Low. The auxotrophic strains contain insertion mutations of TnlOdCamMCS, a transposition defective, chloramphenicol resistant derivative of $\operatorname{Tn} 10$ (14).

\section{Preparation of chromosomal DNA and digestion with SfiI}

Chromosomal DNA was prepared from overnight cultures in $0.8 \%$ InCert agarose (FMC) as described (14). Most commonly, cells were embedded in the agarose plugs at a final cell density equal to $(1 \times)$ or half that $(0.5 \times)$ of the overnight culture $\left(\mathrm{OD}_{600}=6\right.$ for a typical overnight culture). Sometimes a higher DNA concentration was needed (e.g., to examine small DNA fragments or to examine fragments resulting from inefficient cleavage by $S f i I)$. In these cases, the plugs were prepared with a final density of $2.5 \times$, or in one case $10 \times$, relative to the culture.

SfiI enzyme was obtained from Promega. Digestion of chromosomal DNA was carried out in the agarose plugs using the reaction buffer supplied by Promega, with BSA included at the suggested concentration. A sample with a volume of approximately $20 \mu \mathrm{l}$ was digested with 40 units of enzyme at $50^{\circ} \mathrm{C}$ overnight to achieve complete digestion.

For one experiment, cosmid DNA was digested with SfiI (Promega) and either PstI or BamHI (both from Boehringer). Digestions were carried out in a volume of 10 to $20 \mu \mathrm{l}$ with 10 units of enzyme for one hour. SfiI digestions were incubated at $50^{\circ} \mathrm{C}$ and $P s t \mathrm{I}$ and $B a m \mathrm{HI}$ digestions were incubated at $37^{\circ} \mathrm{C}$.

\section{Pulsed field and standard gel electrophoresis and} determination of fragment sizes

Samples were subjected to CHEF pulsed field gel electrophoresis (15) in SeaPlaque agarose gels using the DRII apparatus from Bio-Rad as previously described (14). Typical pulse ramping for standard gels was from 5 to 40 seconds (see figure 1). To increase the resolution of large bands (i.e., larger than $300 \mathrm{~kb}$ ), 75 to 100 second pulse time ramping was used. Bands smaller than $50 \mathrm{~kb}$ were best resolved with 10 to 20 second pulse ramping for 10 hours, followed by 0.5 to 10 second ramping for an additional 16 hours. Fragment sizes were determined by measurements of photographed gels (14). $\lambda$ DNA concatemers were used as size standards for fragments larger than $48.5 \mathrm{~kb}$. Fragments from $10-\mathrm{kb}$ to $48-\mathrm{kb}$ were measured by comparison with a ladder constructed from $\lambda$ DNA digested with several restriction enzymes, giving fragments ranging from $10.1 \mathrm{~kb}$ to $48.5 \mathrm{~kb}$. For the two fragments smaller than $10 \mathrm{~kb}, 1-\mathrm{kb}$ ladder (BRL) was used (unit size, 1,018 bp).
For one experiment, cosmid DNA was analyzed using standard agarose gel electrophoresis. Gels were prepared with $0.6 \%$ agarose (Bio-Rad) in $1 \times \mathrm{TBE}(0.9 \mathrm{M}$ Tris- $\mathrm{HCl}(\mathrm{pH} 7.6), 0.9$ $\mathrm{M}$ boric acid and $1.0 \mathrm{mM}$ EDTA) and electrophoresed at room temperature using standard techniques. The sizes of bands were determined using 1-kb ladder (BRL) as a size standard.

Hybridization of SfiI fragment probes to $E$. coli Gene Mapping Membranes

SfiI fragments were mapped with respect to the genomic restriction map of Kohara et al. $(2,3)$ by hybridizing ${ }^{32} \mathrm{P}$ labelled fragment probes to the $E$. coli Gene Mapping Membrane from Takara Biochemicals (6). The Gene Mapping Membrane contains DNA from each of 476 phage $\lambda$ clones (the miniset of the Kohara library) arranged in a grid. A radiolabelled genomic probe will hybridize to a series of the clones and thus its location on the genomic restriction map can be determined. Specific conditions for the labelling of probes, hybridization to filters and washing and stripping of filters have been described (14). Radiolabelled probe was visualized either by exposing the hybridized and washed filters to $\mathrm{x}$-ray film overnight or by analyzing the filters for one hour using the Betascope 603 Blot Analyzer (Betagen).

\section{Southern blots of CHEF gels}

In one instance a Southern blot of a pulsed field gel of MG1655 chromosomal DNA digested with SfiI was probed with cosmid clone DNA. The methods for the preparation of the filters, the labelling of the probe and the hybridization of the probe to the membrane have been described (14). The pLAFRx cosmid vector and the $E$. coli genomic libraries developed with this vector have also been described (14).

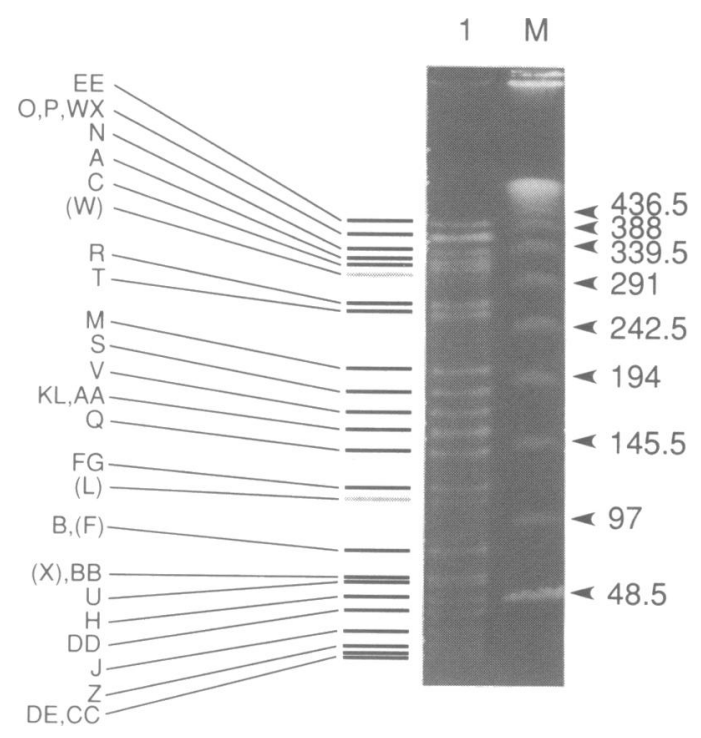

Figure 1. Pulsed field gel of MG1655 digested with Sfil. Lane M: $\lambda$ concatemers. Lane 1: MG1655 digested with SfiI. Bands are labelled according to the letters assigned in figure 2 . Note especially that bands $L$ and $W$ are lighter than surrounding bands due to incomplete cutting at $\mathrm{dcm}$-modified sites. Additional light bands can be seen on gels run under different conditions. The hybrid bands formed due to incomplete cutting are labelled DE, FG, KL and WX. Fragments not visible in this gel are: $\mathrm{K}(46 \mathrm{~kb}), \mathrm{G}(35 \mathrm{~kb}), \mathrm{D}(18 \mathrm{~kb}), \mathrm{E}(10 \mathrm{~kb}), \mathrm{Y}(6$ $\mathrm{kb})$ and I $(3.7 \mathrm{~kb})$. Electrophoresis conditions for this gel were: $1.2 \%$ SeaPlaque agarose in $0.5 \mathrm{x} \mathrm{TBE}, 12^{\circ} \mathrm{C}, 200$ volts, 5 to 40 second pulse ramping for 40 hours. 


\section{Assignment of physical map coordinates using EcoSeq5 and} EcoMap5

The EcoSeq5 E. coli sequence database and the EcoMap5 combined physical and genetic map are updated versions of tools developed by Rudd, et al. at the National Center for Biotechnology Information $(4,5)$. They were obtained by anonymous FTP from the Internet computer ncbi.nlm.nih.gov. EcoSeq5, EcoMap5 and related analysis software were implemented locally on a DEC MicroVax 3900 as detailed in an earlier work (14). The 'Find' program from the Genetics Computer Group Sequence Analysis Software Package, version 7.0 (16) was used to locate $S$ fiI cleavage sites in $E$. coli chromosomal sequences from the EcoSeq5 sequence database. This database contains approximately $38 \%$ of the sequence of the $E$. coli chromosome.

Physical map coordinates for Sfil sites and transposon insertions were assigned based on the coordinate system of EcoMap5 using techniques described previously (14). EcoMap5 is a revised version of the genomic restriction map of Kohara et al. Approximately $37 \%$ of EcoMap5 is based on sequence data, with the remainder being based on the Kohara restriction map. Another $1 \%$ of the chromosome is sequenced, but could not be unambiguously placed on the map. The estimated size of the $E$. coli chromosome based on EcoMap5 is $4673.6 \mathrm{~kb}$.

\section{RESULTS AND DISCUSSION}

Techniques used in the construction of the SfiI map of $E$. coli K-12 strain MG1655

Several approaches were combined in the construction of the SfiI genomic cleavage map of MG1655. These included the measurement of Sfil fragment sizes from CHEF pulsed field gels; the physical analysis of a series of auxotrophic transposon insertion mutations; the placing of Sfil fragments on the genomic map of Kohara, et al.; the searching of sequenced regions of the E. coli chromosome for SfiI recognition sites; and the correlation of all of the above data with the combined genetic and physical map of the $E$. coli genome developed by Rudd and coworkers $(4,5)$.

Measurement of SfiI restriction fragments. With appropriate DNA concentration and pulse time ramping, bands ranging in size from $3.7 \mathrm{~kb}$ to $407 \mathrm{~kb}$ can be visualized on pulsed field gels of MG1655 digested with Sfil. Because special conditions are necessary to visualize and resolve the largest and smallest fragments, it is not possible to see all of these fragments on one gel. For example, it is necessary to prepare DNA at high $(2.5 \times)$ concentration and run the gel with very short pulse times to see the $3.7-\mathrm{kb}$ and $6-\mathrm{kb}$ fragments. An example of a typical gel is shown in figure 1. Fragment sizes are listed in table 1. We have not observed fragments smaller than $3.7 \mathrm{~kb}$ on these pulsed field gels, even when using very concentrated DNA $(10 \times)$. However, there remains a possibility that smaller fragments could have been missed. We feel that fragments in this small size range, should they exist, would have very little impact on the utility of the SfiI map.

In our experience, careful analysis of appropriately prepared gels allows the determination of fragment sizes with a precision of a few percent. A rough estimate of accuracy can be deduced by comparing the predicted chromosome length using Sfi fragment sizes with that predicted by EcoMap5. EcoMap5 predicts a total genome size of $4,673.6 \mathrm{~kb}$ for a $\lambda^{-} \mathrm{F}^{-} \mathrm{K}-12$ strain. Total SfiI fragment sizes, as reported in this work, predict a $4,557 \mathrm{~kb}$ chromosome-a difference of $2.6 \%$.
The measurement of small fragments deserves special consideration. For fragments smaller than $48 \mathrm{~kb}$, we experimented with several size standards. These included a 10 to $48 \mathrm{~kb}$ size standard derived from $\lambda$, the $1-\mathrm{kb}$ ladder from BRL

Table 1. Summary of evidence for fragment placement on the SfiI map of MG1655.

\begin{tabular}{|c|c|c|c|c|}
\hline Fragment & Size $(\mathbf{k b})$ & $\begin{array}{l}\text { Transposon } \\
\text { Insertions }\end{array}$ & $\begin{array}{l}\text { Sequenced } \\
\text { Endpoints }^{\mathrm{a}}\end{array}$ & $\begin{array}{l}\text { Gene Mapping } \\
\text { Membrane } \\
\text { Probed }\end{array}$ \\
\hline A & 326 & $\operatorname{car} A B, \operatorname{nad} C$ & $\mathbf{u}$ & + \\
\hline B & 79 & lacZ & & \\
\hline C & 312 & & d & + \\
\hline$D^{b}$ & 18 & & $\mathbf{u}$ & $t^{c}$ \\
\hline $\mathrm{E}^{\mathrm{b}}$ & 10 & & & $t^{c}$ \\
\hline $\mathrm{F}^{\mathrm{b}}$ & 79 & galETK $^{\mathrm{d}}$ & d & \\
\hline$G^{b}$ & 35 & galETK $^{\mathrm{d}}$ & u & \\
\hline $\mathrm{H}^{\mathrm{e}}$ & 54 & & d & \\
\hline $\mathrm{I}^{\mathrm{f}}$ & 3.7 & & $\mathrm{u}, \mathrm{d}$ & + \\
\hline $\mathbf{J}$ & 36 & aroA & $\mathrm{u}$ & + \\
\hline $\mathrm{K}^{\mathrm{b}}$ & 46 & pyrD & d & $+g$ \\
\hline $\mathrm{L}^{\mathrm{b}}$ & 109 & pyrC & u & $\mathrm{g}$ \\
\hline $\mathbf{M}$ & 203 & $\operatorname{trp} E D C B A$ & & \\
\hline $\mathbf{N}$ & 343 & & d & + \\
\hline 0 & 370 & aroD & u & + \\
\hline $\mathbf{P}$ & 370 & $\begin{array}{l}\text { hisGDCBHAFI, } \\
\text { purF }\end{array}$ & d & + \\
\hline Q & 139 & & u & + \\
\hline $\mathbf{R}$ & 268 & $\begin{array}{l}\text { purC, guaBA, } \\
\text { purL, pheA, tyrA }\end{array}$ & d & \\
\hline $\mathbf{S}$ & 183 & cysHIJ & $\mathbf{u}$ & \\
\hline $\mathrm{T}$ & 256 & serA, galP & d & \\
\hline $\mathrm{U}$ & 60 & $\arg G$ & $\mathrm{u}, \mathrm{d}$ & + \\
\hline V & 166 & & $\mathrm{u}, \mathrm{d}$ & + \\
\hline $\mathbf{W}^{\mathrm{b}}$ & 300 & malPQT & $\mathrm{u}, \mathrm{d}$ & $t^{h}$ \\
\hline $\mathrm{X}^{\mathrm{b}}$ & 63 & & $\mathrm{u}, \mathrm{d}$ & $+^{h}$ \\
\hline $\mathrm{Y}$ & 6 & & $\mathrm{u}, \mathrm{d}$ & + \\
\hline $\mathrm{Z}$ & 28 & & $\mathrm{u}, \mathrm{d}$ & + \\
\hline AA & 152 & $m e t E$ & $\mathbf{u}$ & \\
\hline BB & 63 & & d & + \\
\hline $\mathrm{CC}$ & 25 & & $\mathrm{u}, \mathrm{d}$ & + \\
\hline DD & 47 & & $\mathrm{u}, \mathrm{d}$ & + \\
\hline $\mathrm{EE}$ & 407 & thrABC & $\mathrm{u}, \mathrm{d}$ & \\
\hline
\end{tabular}

${ }^{a} d$ : downstream endpoint is sequenced; $u$ : upstream endpoint is sequenced (upstream and downstream are used in reference to a clockwise progression around the map).

bThere are four known Sfil recognition sites that are affected by $\mathrm{dcm}$ methylation. Since Sfi appears to cleave these sites very inefficiently, larger hybrid fragments due to incomplete cleavage are observed. Specifically, hybrid fragment DE migrates as a doublet with 28-kb fragment $\mathrm{Z}$; hybrid fragment FG migrates at $118 \mathrm{~kb}$; hybrid fragment $\mathrm{KL}$ migrates as a doublet with $152-\mathrm{kb}$ fragment AA; and hybrid fragment WX migrates as a triplet with $370-\mathrm{kb}$ fragments $\mathrm{O}$ and $\mathrm{P}$. ${ }^{c}$ Fragment $\mathrm{D}$ and hybrid fragment DE $(25 \mathrm{~kb})$ were used as Gene Mapping Membrane probes. Fragment $\mathrm{E}$ was placed by inference from these results.

${ }^{\mathrm{d}}$ The galETK::Tn1OdCamMCS insertion is seen to affect both fragment $\mathrm{F}$ and hybrid fragment FG; and Fragment $\mathrm{F}$ was placed by hybridization to a Gene Mapping Membrane. Fragment $G$ was placed by inference from these results. ${ }^{\mathrm{e}} \mathrm{A}$ cosmid clone carrying the aroA gene hybridized to both fragments $\mathrm{H}$ and $\mathrm{J}$ when it was used to probe a Southern blot of an Sfil digest of MG1655. Since fragment $J$ was placed by hybridization to the Gene Mapping Membrane, this constrained the position of fragment $\mathrm{H}$ to the other side of the aroA locus.

${ }^{f}$ Fragment $I$ is completely contained within two cosmids complementing the aroA::TnlOdCamMCS insertion. A fragment with the same mobility as the cloned fragment was noted on some CHEF gels. The equivalence of the cloned and uncloned fragments was confirmed by hybridization of the uncloned fragment to a Gene Mapping Membrane.

${ }^{\mathrm{g}}$ Fragments $\mathrm{KL}$ (152 kb), $\mathrm{K}$ and $\mathrm{L}$ were all used as Gene Mapping Membrane probes.

${ }^{h}$ Fragments WX ( $\left.370 \mathrm{~kb}\right), \mathrm{W}$ and $\mathrm{X}$ were all used as Gene Mapping Membrane probes.

'The malPQ and malT cistrons are close to the Sfil cleavage site separating fragments $\mathbf{V}$ and $\mathbf{W}$. Therefore, a strain with an insertion in this position does not show any differences in fragment size when compared to wild type. 
(unit size 1,018 bp), and the 5-kb ladder from Bio-Rad consisting of concatemers of linearized pBR328 plasmid (unit size, $4.9 \mathrm{~kb}$ ). On pulsed field gels run under small fragment conditions (see Materials and Methods), the standards were found to be inconsistent with each other. For instance, the 4.9-kb and 19.6-kb bands from the 5-kb ladder migrated with the 1-kb ladder's 4-kb and 12-kb bands, respectively. In the case of fragment $\mathrm{Y}$, whose exact length is known from sequence data, the $1-\mathrm{kb}$ ladder gave more accurate measurements. The discrepancy between different size standards disappeared when the markers were run on standard agarose gels. These observations indicate that, under the conditions used for small fragments, migration of DNA in CHEF pulsed field agarose gel electrophoresis has a significant sequence dependence. One explanation for this phenomenon is that DNA secondary structures such as static DNA bends may influence migration patterns in CHEF pulsed field gels. These structures are known to influence migration in other electrophoretic methods (17). This would be expected to be more severe for smaller fragments. For consistency, we used the $\lambda$ derived ladder for fragments from $10 \mathrm{~kb}$ to $48 \mathrm{~kb}$, and the $1-\mathrm{kb}$ ladder for fragments smaller than $10 \mathrm{~kb}$. However, it should be noted that the accuracy of these measurements may be somewhat compromised.

Auxotrophic transposon insertion mutants. Once the sizes of the wild type fragments were determined, SfiI digests of DNA from each of twenty-six different auxotrophic transposon insertion mutants were subjected to pulsed field gel electrophoresis. The TnlOdCamMCS transposon contains an Sfil cleavage site to allow for its precise mapping (14). Similar approaches to the mapping of wild type transposon insertions have been taken by others (e.g., 18). All twenty-six insertions have previously been mapped to specific genetic loci (14). Most insertions occur in genes that are sequenced and placed on EcoMap5, including the phe $A$ and tyr $A$ genes that are newly-placed in this release of EcoMap. This placement allows the insertions to be assigned physical coordinates. The few target genes that have not been sequenced can be placed on the physical map to within a few kilobases by their location relative to nearby placed genes. Figure 2 shows the locations of the transposon insertions and the genetic loci to which they have been mapped.

If a particular SfiI fragment contains a TnlOdCamMCS insertion, a new chromosomal Sfil site is generated at the insertion site. When the SfiI restriction pattern is compared to that of wild type, the target fragment disappears and is replaced by two smaller bands. By measuring the sizes of these two new bands, the transposon insertion can be accurately placed within the fragment. Since the insertion is, in turn, placed on the genetic and physical maps, the fragment can be localized on these maps.

If a fragment contains only one transposon insertion, its orientation with respect to the insertion cannot be determined by this data alone. Additional data, such as another insertion in this fragment, or the existence of adjacent placed fragments or adjacent sequenced SfiI sites, can serve to constrain the fragment to only one of the two possible positions. In all, sixteen of the thirty-one Sfil fragments contained at least one insertion (see table 1 and figure 2).

In two cases, that of the aro $A:: T n l O \mathrm{dCamMCS}$ insertion in fragment $\mathrm{J}$ and that of the malPQT::Tn10dCamMCS insertion in fragment W, the insertions were very close to wild type SfiI sites. In the case of fragment $\mathrm{W}, \mathrm{a} 300-\mathrm{kb}$ fragment, the insertion caused no discernible change in fragment size, and $\mathrm{W}$ could not be placed based on this insertion. In the case of fragment $\mathrm{J}$, a 36-kb fragment, the insertion of the 1-kb TnlOdCamMCS transposon caused a noticeable increase in the size of the larger daughter fragment when compared to the wild type parent fragment. This increase in size allowed the placement of fragment $\mathrm{J}$.

Mapping of fragments to the Kohara physical map. After all fragments with transposon insertions had been placed by the above method, over half of the fragments remained unplaced. To place these fragments, a new physical mapping tool, the $E$. coli Gene Mapping Membrane from Takara Biochemicals, was used. A membrane was probed with a labelled SfiI restriction fragment allowing the fragment to be localized on the physical map of Kohara et al. (2). Since the EcoMap5 physical map is based on the Kohara map, coordinates for fragment endpoints can be accurately assigned based on this data. The Gene Mapping Membranes were probed with a total of twenty-one fragments, providing evidence for twenty-three of the thirty-one SfiI restriction sites (see tables 1 and 2).

An interesting observation was made when the membranes were probed with fragments containing an rRNA cistron. These probes hybridize not only with their overlapping clones, but also with the clones containing the other six rRNA cistrons. We have not, however, observed the cross-hybridization of smaller regions, such as IS elements.

Sequence database analysis. Since $38 \%$ of the $E$. coli genomic sequence is currently available in EcoSeq5, one would predict that several $S f$ iI restriction sites have been sequenced. Fourteen sequenced Sfil recognition sites have been previously reported $(19,20)$. When EcoSeq5-containing GenBank and EMBL sequences up to 1 December 1991, as well as several unpublished sequences-was searched for SfiI recognition sites, twenty-one sites were found.

Of these twenty-one sequenced sites, two were not cleaved in MG1655. All other sites were confirmed experimentally. One of the uncleaved sites was found in nrdAecoM (access. no. ES1162; also in GenBank sequence ECONRDA, access. no. K02672) (21), upstream of the $n r d A$ gene. If cleaved completely, the site in this sequence would cause the disappearance of the $370-\mathrm{kb}$ fragment $\mathrm{P}$ and the appearance of a $275-\mathrm{kb}$ and a $95-\mathrm{kb}$ fragment. The other uncleaved site is present in yidC (EcoSeq5 sequence gyrBecoM, access. no. ES1230; also in unpublished sequence rnpAeco, access. no. ES3214) (22). Cleavage at this site would divide the $63-\mathrm{kb}$ fragment $\mathrm{X}$ into two fragments, each approximately $32 \mathrm{~kb}$ in size. None of the predicted products were noted on pulsed field gels. Therefore, we are confident that these sites are not cleaved to any significant degree in MG1655. There are a few possible explanations for these discrepancies. One, it is possible that these sequences contain errors and that the particular strains whose DNA was sequenced did not contain SfiI recognition sites at these locations. Another explanation is that these discrepancies represent bona fide sequence differences between MG1655 and the sequenced strain. A third possibility is that these sites are present in MG1655 but are not cleaved for some reason (see discussion of methylation-sensitive sites below).

A comparable rate of disagreement between the sequence databases and experimental evidence was observed in the NotI map of MG1655 (14). This map showed one discrepancy out of eleven sequenced cleavage sites. Taken together, the SfiI and NotI data show a frequency of disagreement between sequence data and experimental evidence in MG1655 of ten percent. This 
disagreement between published sequence and experimental data underscores the importance of experimental evidence in the construction of physical maps.

Two of the sequenced sites, sS25 and sS26, occur in the same sequence-namely, bglBecoM (see Table 2) (23). This allows precise prediction of the size of the fragment bounded by these sites as 6,017 base pairs. Our experimental data confirm the placement of an appropriately sized fragment in this region of the physical map.
Placement of $3.7 \mathrm{~kb}$ fragment $\mathrm{I}$. Because of its small size, the placement of fragment I presented a special challenge. A very faint fragment of about $3.7 \mathrm{~kb}$ was visualized on some high concentration, short pulse time gels. However, even when using DNA made at very high concentration (10x relative to the density of the overnight culture), labelling of fragment I with ${ }^{32} \mathrm{P}$ produced marginal results. This is not surprising considering that a $3.7-\mathrm{kb}$ fragment represents less than $0.08 \%$ of the total DNA. Our best efforts produced only a questionable hybridization to

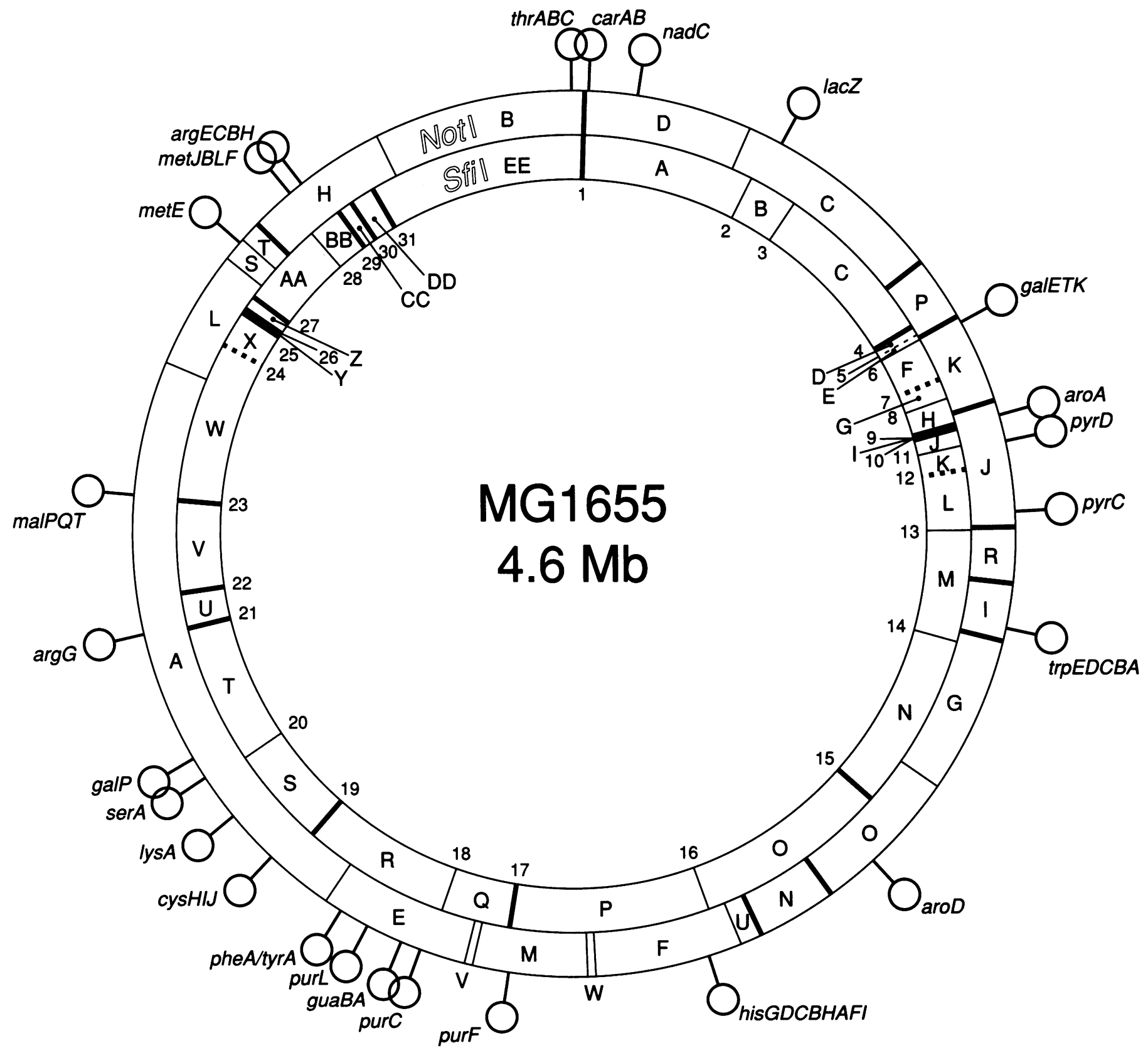

Figure 2. Sfi and NotI restriction maps of Escherichia coli strain MG1655. The map is oriented so that zero minutes on the genetic map is located at the top at locus thrABC. Sfi fragments (inner circle) are lettered consecutively from A to EE beginning at site sS1 (25.1 kb) near 0 minutes and continuing in a clockwise direction. The NotI map of MG1655 (outer circle) was described previously (14). Sfil sites are also numbered on the inside of the map in a clockwise orientation from zero minutes. Transposon insertion locations are designated by lollipops and are labelled with the inactivated genetic locus. Sfil and NotI sites present in sequenced regions of the chromosome are designated with thick lines. Sfil sites present in unsequenced regions are designated with thin lines. Sequenced SfiI sites containing an internal $\mathrm{dcm}$ methylation site (sS7, sS12, and sS24) are designated with thick dashed lines. The unsequenced $d \mathrm{~cm}$-sensitive site (sS5) is labeled with a thin dashed line. These four sites are differentiated from other sites due to their very inefficient cleavage in MG1655 $\left(\mathrm{dcm}^{+}\right)$. 
1134 Nucleic Acids Research, Vol. 20, No. 5

Table 2. Physical and genetic map locations of Sfil cleavage sites

\begin{tabular}{|c|c|c|c|c|c|c|}
\hline Feature & $\begin{array}{l}\text { Location on } \\
\text { physical } \\
\operatorname{map}(\mathrm{kb})^{\mathrm{a}}\end{array}$ & $\begin{array}{l}\text { Location in Kohara } \\
\text { phage miniset }\end{array}$ & $\begin{array}{l}\text { Location on genetic } \\
\text { map (minutes) }\end{array}$ & Gene & $\begin{array}{l}\text { Database sequence \& } \\
\text { position in sequence }\end{array}$ & Evidence $^{\mathrm{c}}$ \\
\hline $\mathrm{sS} 1$ & 25.093 & {$[103] 22 \mathrm{~B} 12$} & 0.4 & ileS & $\begin{array}{l}\text { dnaKecoM (ES1244) } 12794 \\
\text { ileSeco }(E S 3048)^{d} 2380\end{array}$ & a $S t$ \\
\hline sS2 & $360 \pm 2$ & $\begin{array}{l}{[137] 9 \mathrm{~F} 1} \\
{[138] 10 \mathrm{~A} 6}\end{array}$ & 7.8 & & & a G S t \\
\hline sS3 & $449 \pm 3$ & $\begin{array}{l}{[146] 19 \mathrm{~F} 6} \\
{[147] 2 \mathrm{H} 5}\end{array}$ & $9.6 \pm 0.1$ & & & a G t \\
\hline sS4 & 762.961 & {$[176] 7 \mathrm{E} 10$} & 16.3 & upstream of $g / t A$ & $\begin{array}{l}\text { gltAecoM (ESI058) } 663 \\
\text { ECOGLTA (J01619) } 663\end{array}$ & g S \\
\hline $\mathrm{sS}^{\mathrm{c}}$ & $780 \pm 1$ & $\begin{array}{l}{[177] 18 \mathrm{~F} 11} \\
{[178] 10 \mathrm{G} 5}\end{array}$ & 16.6 & & & a G S \\
\hline sS6 & $791 \pm 1$ & $\begin{array}{l}{[178] 10 \mathrm{G5}} \\
{[179] 4 \mathrm{HI}}\end{array}$ & 16.8 & & & a G S t \\
\hline $\mathrm{sS} 7^{\mathrm{e}}$ & 873.860 & $\begin{array}{l}{[207] 3 \mathrm{E} 5} \\
{[208] 1 \mathrm{E} 2}\end{array}$ & 18.2 & $\operatorname{chlN}$ & ECOCHLEN (M21151) 1707 & g S \\
\hline sS8 & $909 \pm 1$ & {$[211] 14 \mathrm{H} 6$} & 18.8 & & & A $\mathrm{T}$ \\
\hline sS9 & 962.927 & $\begin{array}{l}{[216] 13 \mathrm{E} 3} \\
{[217] 6 \mathrm{D} 12}\end{array}$ & 20.0 & upstream of $y c a E$ & $\begin{array}{l}\text { pfl-ecoM (ES1221) } 4348 \\
\text { M26413 (M26413) } 525\end{array}$ & a c S \\
\hline sS10 & 966.554 & $\begin{array}{l}{[216] 13 \mathrm{E} 3} \\
{[217] 6 \mathrm{D} 12}\end{array}$ & 20.2 & $\operatorname{ser} C$ & ECOAROA (X00557) 932 & a c S t \\
\hline sS11 & $1003 \pm 1$ & $\begin{array}{l}{[220] 7 \mathrm{~F} 6} \\
{[221] 9 \mathrm{E} 9}\end{array}$ & $21.1 \pm 0.1$ & & & a $G t$ \\
\hline $\mathrm{sS} 12^{\mathrm{e}}$ & 1048.899 & $\begin{array}{l}{[224] 4 \mathrm{H} 9} \\
{[225] 4 \mathrm{H} 11}\end{array}$ & 22.5 & appA & $\begin{array}{l}\text { appAecoM (ES1006) } 538 \\
\text { ECOAPPAA (M58708) } 363\end{array}$ & $\mathrm{~g} \mathrm{~S}$ \\
\hline sS13 & $1162 \pm 2$ & $\begin{array}{l}{[235] 14 \mathrm{Cl}} \\
{[236] \mathrm{E} 9 \mathrm{G} 1}\end{array}$ & 24.7 & & & a G t \\
\hline sS14 & $1368 \pm 2$ & $\begin{array}{l}{[256] \mathrm{E} 14 \mathrm{~F} 6} \\
{[257] 1 \mathrm{C} 2}\end{array}$ & $29.3 \pm 0.1$ & & & a G S t \\
\hline sS15 & 1708.725 & $\begin{array}{l}{[314] 6 \mathrm{~A} 5} \\
{[315] 9 \mathrm{Al}}\end{array}$ & 35.8 & $\operatorname{malX}$ & $\begin{array}{l}\text { mallecoM (ES1168) } 2927 \\
\text { ECOMALAA (M60722) } 1986\end{array}$ & $\mathrm{~g} \mathrm{~S} \mathrm{t}$ \\
\hline sS16 & $2081 \pm 1$ & $\begin{array}{l}{[347] 9 \mathrm{~B} 2} \\
{[348] 3 \mathrm{~B} 12}\end{array}$ & $43.6 \pm 0.2$ & & & a $\mathrm{S} \mathrm{T}$ \\
\hline $\mathrm{sS} 17$ & 2459.300 & $\begin{array}{l}{[407] 12 \mathrm{~F} 12} \\
{[408] 2 \mathrm{G} 11}\end{array}$ & 50.3 & $f a b B$ & ECOFABB (M24427) 600 & $\mathrm{~g} \mathrm{~S} \mathrm{t}$ \\
\hline sS18 & $2592 \pm 2$ & {$[422] 7 \mathrm{~A} 8$} & 53.0 & & & a G T \\
\hline sS19 & 2875.550 & $\begin{array}{l}{[449] 12 \mathrm{~F} 2} \\
{[450] 25 \mathrm{D} 2}\end{array}$ & 58.7 & mutS & $\begin{array}{l}\text { hycHecoM (ES1158) } 14832 \\
\text { ECOMUTS (M64730) } 1252\end{array}$ & $S \mathrm{t}$ \\
\hline sS20 & $3066 \pm 2$ & $\begin{array}{l}{[469] 5 \mathrm{E} 11} \\
{[470] 10 \mathrm{~B} 4}\end{array}$ & $62.5 \pm 0.2$ & & & a $\mathrm{T}$ \\
\hline sS21 & 3330.452 & $\begin{array}{l}{[518] 13 \mathrm{~A} 9} \\
{[519] \mathrm{E} 4 \mathrm{G} 11 \mathrm{~S}}\end{array}$ & 68.8 & $\begin{array}{l}\text { between } r p s O \\
\text { and pnp }\end{array}$ & $\begin{array}{l}\text { mtr-ecoM (ESII } 184) 7454 \\
\text { ECORPSOP (J02638) } 441\end{array}$ & a $S \mathrm{t}$ \\
\hline $\mathrm{sS} 22$ & 3395.498 & $\begin{array}{l}{[525] 8 D 6} \\
{[526] 1 \mathrm{E} 3}\end{array}$ & 70.3 & $\operatorname{ssp} B$ & $\begin{array}{l}\text { sspBecoM (ES1200) } 2623 \\
\operatorname{sspBeco}_{(\mathrm{ES} 3156)^{\mathrm{d}}} 509\end{array}$ & a g S t \\
\hline sS23 & 3571.304 & $\begin{array}{l}{[619] \mathrm{E} 3 \mathrm{C} 10} \\
{[618] \mathrm{E} 4 \mathrm{E} 4}\end{array}$ & 75.3 & malQ & $\begin{array}{l}\text { malQecoM (ESI072) } 1408 \\
\text { ECOMALQP (M32793) } 1447\end{array}$ & a g S t \\
\hline $\mathrm{sS} 24^{\mathrm{e}}$ & 3876.486 & $\begin{array}{l}{[569] 1 \mathrm{D} 11} \\
{[568] 7 \mathrm{C} 4} \\
{[567] 8 \mathrm{E} 8}\end{array}$ & 82.6 & $i l v B$ & $\begin{array}{l}\text { uhpTecoM (ES1135) } 6807 \\
\text { ECOILVBPR (J01633) } 833\end{array}$ & a $\mathrm{g} \mathrm{S}$ \\
\hline sS25 & 3946.882 & {$[560] 2 \mathrm{Al}$} & 84.2 & uncA & $\begin{array}{l}\text { bglBecoM (ES1019) } 17664 \\
\text { ECOUNCC (X01631) } 6483\end{array}$ & a $\mathrm{g} \mathrm{S}$ \\
\hline sS26 & 3952.899 & $\begin{array}{l}{[560] 2 \mathrm{~A} 1} \\
{[559] 4 \mathrm{~F} 4}\end{array}$ & 84.2 & gidA & $\begin{array}{l}\text { bglBecoM (ES1019) } 23681 \\
\text { ECOUNCC }(\mathrm{X} 01631) 466\end{array}$ & a $g \mathrm{~S}$ \\
\hline sS27 & 3981.219 & $\begin{array}{l}{[558] 15 \mathrm{D} 10} \\
{[557] 2 \mathrm{E} 6} \\
{[556] 2 \mathrm{~A} 8}\end{array}$ & 84.9 & $i l v E$ & $\begin{array}{l}\text { rrnCecoM (ESI227) 12163 } \\
\text { ECOILVGE (M10313) } 3112\end{array}$ & a g S t \\
\hline sS28 & $4143 \pm 1$ & $\begin{array}{l}{[540] 8 \mathrm{~B} 10} \\
{[539] 4 \mathrm{H} 12}\end{array}$ & 88.8 & & & A G T \\
\hline sS29 & 4208.466 & none $^{f}$ & 89.9 & $r p l A$ & $\begin{array}{l}\text { oxyRecoM (ES1145) } 20968 \\
\text { ECORPLRPO (J01678) } 976\end{array}$ & a g S \\
\hline sS30 & 4237.463 & $\begin{array}{l}{[532] 9 B 9} \\
{[531] 3 \mathrm{C} 5}\end{array}$ & 90.4 & purH & $\begin{array}{l}\text { hydGecoM (ES1148) } 4365 \\
\text { ECOPURHD (J05126) } 541\end{array}$ & a $g \mathrm{~S}$ \\
\hline sS31 & 4284.786 & $\begin{array}{l}{[634] 1 \mathrm{~F} 8} \\
{[635] 12 \mathrm{~B} 4}\end{array}$ & 91.8 & $p l s B$ & $\begin{array}{l}\text { plsBecoM (ES1094) } 2288 \\
\text { ECOPLSB (K00127) } 1566\end{array}$ & a g S t \\
\hline
\end{tabular}

${ }^{\mathrm{a} C}$ Coordinates based on EcoMap5. The beginning of the $t h r$ operon is defined as $0 \mathrm{~kb}(0 \mathrm{~kb}=0$ minutes).

${ }^{b}$ EcoSeq5 sequences are listed first. In the case of melds, the component sequence that contains the SfiI site is listed below the EcoSeq5 meld. Accession numbers are given in parentheses. Positions refer to the beginning of the SfII recognition site with respect to the beginning of the sequence. Sequences in all caps are also present in GenBank.

${ }^{c}$ Evidence used to assign coordinates to the sites. Upper case letters represent evidence used to assign the coordinate or coordinate range to the site. Lower case letters represent evidence that supports this coordinate or range. A/a: location of site deduced from the location of adjacent sites whose location was directly determined: $\mathbf{c}$ : site confirmed by digesting a cosmid clone containing the site with Sfil; G/g: location of site determined by hybridization of an MGI655 Sfil fragment to a Gene Mapping Membrane; S: site is present in a published DNA sequence which has been placed on EcoMap5, or a placed sequence without any SfiI sites restricts the location of a site to outside of the sequenced area; T/t: location of site deduced by analyzing Sfil digests of strains containing mapped transposon insertions.

${ }^{\mathrm{d}}$ The sequences ileSeco and sspBeco are unpublished sequences that are unique to EcoSeq5.

${ }^{\mathrm{e}}$ Sites sS7, sS12 and sS24 are sequenced and contain an internal $\mathrm{dcm}$ methylation site that causes digestion by Sfil to be very inefficient. sS5 is unsequenced but behaves consistently with having a similar internal $\mathrm{dcm}$ site overlap.

Site sS29 is located in a $6.5 \mathrm{~kb}$ gap between miniset clones [534]E11C11 and [533]7B7. 
miniset clones [216]13E3 and [217]6D12. This would place the fragment between $960 \mathrm{~kb}$ and $970 \mathrm{~kb}$ on EcoMap5. This placement is consistent with two sequenced sites separated by $3.7 \mathrm{~kb}$ in this region of the map (see Table 2). Direct confirmation of this placement was provided by digestion with SfiI of two MG1655-derived cosmids that complement a nearby aroA mutation. The physical map locations of these cosmids were determined by comparing the PstI and BamHI patterns of the inserts with those present in EcoMap5 in the region of aroA. By this map placement, both cosmids were predicted to contain the entire 3.7-kb SfiI fragment. Digestion of the cosmids with $S$ SiI confirmed this and the $3.7-\mathrm{kb}$ fragment I was clearly seen on both a standard gel and a pulsed field gel.

Alignment with EcoMap5. To assign specific physical coordinates to $S$ fiI recognition sites, the sequence-modified version of the Kohara physical map, EcoMap5, was used as a standard. The predicted total length of the genome differs by $117 \mathrm{~kb}$ between EcoMap5 and the SfiI map. Nevertheless, this map provides a convenient framework for locating sites and fragments, as well as correlating the map with other physical maps of $E$. coli. Table 2 is a listing of the SfiI sites, their EcoMap5 coordinates and a summary of the evidence for each, as well as genetic and sequence database location information.

The nineteen sequenced sites were easiest to align because the sequences were already placed on EcoMap5. Gene Mapping Membrane experiments provided evidence for nine of the remaining twelve sites. Because of the close association between the Gene Mapping Membranes, the Kohara miniset, and EcoMap5, evidence from Gene Mapping Membrane experiments could place sites on EcoMap5 relatively accurately. The three remaining sites were placed based on transposon insertion data, combined with extrapolation from adjacent sequenced sites and adjacent fragment lengths.

In a few cases, the experimentally determined range for a site overlapped sequenced regions that did not contain an Sfi recognition site. These sequenced regions were used to narrow the site's coordinate range further. In other cases, multiple types of evidence combined to give a narrower range than could have been derived from any one approach. A combination of all of these approaches enabled us to assign EcoMap5 coordinate ranges of plus or minus one to two kilobases to all unsequenced sites except sS3, which has a range of plus or minus three kilobases (table 2).

\section{The map}

Figure 2 depicts the $S f$ il restriction map of $E$. coli $\mathrm{K}-12$, strain MG1655. This map is aligned with the NotI map of the same strain for comparison. Table 1 lists all the SfiI fragments, along with sizes and evidence for their existence and placement on the map. Table 2 summarizes the locations of the thirty-one SfiI cleavage sites, along with the evidence for each site.

Nomenclature. We have recently constructed a NotI restriction map of $E$. coli K-12, strain MG1655 (14) in which we adopted a nomenclature based on the EMG2 map of Smith and colleagues originally published in 1987 (24). Although there were some differences in the two maps, the fragments of the MG1655 map were named to avoid confusion with this widely circulated EMG2 map. Smith and coworkers have also published a list of SfiI fragments of EMG2, along with a partial map of these fragments $(19,24)$. When the partial map was compared with the SfiI map of MG1655 presented here, there were numerous differences affecting over half of the map. In view of these discrepancies, we found it difficult to base the MG1655 nomenclature on that published for EMG2. Therefore, we have adopted a site nomenclature that begins with $\mathrm{sS} 1$ at the $25.1 \mathrm{~kb}$ site (based on EcoMap5) and continues clockwise to sS31. Fragment names begin with $\mathrm{A}$, between sites $\mathrm{s} 1$ and $\mathrm{sS} 2$, and proceed clockwise through EE.

Persistent partial cleavage of a subset of $\mathrm{Sfi} I$ sites. When a pulsed field gel of MG1655 digested with SfiI such as the one shown in figure 1 is examined carefully, there are several bands whose intensity does not seem stoichiometrically related to the surrounding bands. The more intense bands, such as the $370-\mathrm{kb}$ and $152-\mathrm{kb}$ bands, are easily explained as being doublets or triplets. Experimental evidence confirms this explanation. Lighter bands, such as the $300-\mathrm{kb}$ band and the 109-kb band, are typically due to incomplete digestion of the DNA. However, even when digestion with SfiI is carried to completion, the $300-\mathrm{kb}$ and $109-\mathrm{kb}$ bands (and several other bands not visible in figure 1) still appear lighter than surrounding bands.

The reason for these light bands was suggested by the unexpected placement of the $109-\mathrm{kb}$ and $46-\mathrm{kb}$ nonstoichiometric

\begin{tabular}{|c|c|c|c|}
\hline Class & Site & Sequence & fil cleavage \\
\hline I & sS22 & $\begin{array}{l}\text { AAACAGGCCCAGGCGGCCTGTTT } \\
\text { TTTGTCCGGGTCCGCCGGACAAA }\end{array}$ & complete \\
\hline \multirow[t]{3}{*}{ II } & sS7 & $\begin{array}{l}\text { TAGTGGGCCTGGGCGGCCTCGGC } \\
\text { ATCACCCGGACCCGCCGGAGCCG }\end{array}$ & partial \\
\hline & sS12 & $\begin{array}{l}\text { TTACCGGCCAGGTTCCCCATGCG } \\
\text { AATGGCCGGTCCAACCGGTACGC }\end{array}$ & partial \\
\hline & sS24 & $\begin{array}{l}\text { CACCGGGCCTGGGCGGCCTGATT } \\
\text { GTGGCCCGGACCCGCCGGACTAA }\end{array}$ & partial \\
\hline III & - & $\begin{array}{l}\text { ACCAGGGCCGTAClCGCCCAGTGG } \\
\text { TGGTCCCGGCATGGCCCGTCACC }\end{array}$ & none \\
\hline \multirow[t]{2}{*}{ IV } & sS1 & $\begin{array}{l}\text { TGCCACGCCAAACACGCCTTCGT } \\
\text { ACGGTCCGGTTTGTCCGGAAGCA }\end{array}$ & complete \\
\hline & sS15 & $\begin{array}{l}\text { CACCACGCCGACAAAGCCCCATCA } \\
\text { GTGGTCCGGCTGTTCCGGGTAGT }\end{array}$ & complete \\
\hline & sS29 & $\begin{array}{l}\text { AACCTCGCCCAGCTGGCCAACAA } \\
\text { TTGGACCGGGTCGACCGGTTGTT }\end{array}$ & complete \\
\hline & sS30 & $\begin{array}{l}\text { GTCCTEGCCGCGALGGCCCAGAA } \\
\text { CAGGACCGGCGCTGCCGGGTCTT }\end{array}$ & complete \\
\hline \multicolumn{4}{|c|}{$\begin{array}{l}\text { Figure 3. Sequenced SfiI sites containing } d c m \text { methylation sites. Of the twenty } \\
\text { one SfiI sites (GGCCNNNNNGGCC) that are present in sequenced regions o } \\
\text { the chromosome, nine overlap a } d c m \text { methylase recognition site (CCWGG). Thes } \\
\text { can be subdivided into four groups based on the location of the methylas } \\
\text { recognition site relative to the SfiI recognition site as follows: class I, one bas } \\
\text { pair internal overlap; class II, two base pair internal overlap; class III, one bas } \\
\text { pair external overlap; class IV, two base pair external overlap. The nondegenerat } \\
\text { bases of the SfiI recognition site are shown in bold type. The cytosine residue } \\
\text { that are methylated by the } d c m \text { methylase are boxed. A line bisects each sequenc } \\
\text { along the } S \text { fiI cleavage plane. Sequences are taken from EcoSeq } 5 \text {. The class II }\end{array}$} \\
\hline
\end{tabular}
site does not have a site number because it is not cleaved (see text). 
fragments at the same position as a previously mapped 152-kb fragment. This placement was confirmed by both transposon insertion data and Gene Mapping Membrane experiments and indicates that the site separating $\mathrm{K}$ and $\mathrm{L}$ is partially cleaved. This results in both a hybrid fragment and its two component fragments being present in a chromosomal SfiI digestion. There are four such partially-cleaved sites in MG1655. In the above example, fragment $\mathrm{K}(46 \mathrm{~kb})$ and fragment $\mathrm{L}(109 \mathrm{~kb})$ are present in 152-kb hybrid fragment $\mathrm{KL}$, due to partial cleavage of site $\mathrm{sS} 12$. In addition, fragments $\mathrm{D}(18 \mathrm{~kb})$ and $\mathrm{E}(10 \mathrm{~kb})$ are present in the 25-kb hybrid fragment $\mathrm{DE}$, due to partial cleavage of site sS5; fragments $F(79 \mathrm{~kb})$ and $\mathrm{G}(35 \mathrm{~kb})$ are present in the $118-\mathrm{kb}$ hybrid fragment FG, due to partial cleavage of site sS7; and fragments W (63 kb) and X (300 kb) are present in 370-kb hybrid fragment WX, due to partial cleavage of site sS24.

Three of the four inefficiently cleaved sites are sequenced (sS7, $\mathrm{sS} 12$ and sS24). An examination of these sequenced sites led to the observation that, in all three cases, the SfiI recognition sequence GGCCNNNNNGGCC overlaps the recognition sequence for the $\mathrm{dcm}$ methylase, CCWGG. Furthermore, out of the twenty-one sequenced SfiI sites, six more sites, for a total of nine, overlap $\mathrm{dcm}$ methylation sites. Of these six additional $d c m-S f i$ sites, five-sS1, sS15, sS22, sS29 and sS30-appeared to be completely cleaved. One, present in the EcoSeq5 sequence rnpAeco (22), did not appear to be cleaved at all (see discussion of sequence database analysis, above). To examine the effect of $\mathrm{dcm}$ methylase activity on the efficiency of SfiI cleavage, we examined the restriction pattern of a K-12 strain lacking $\mathrm{dcm}$ methylase activity. The pattern was similar to that of MG1655 with the exception that the four hybrid fragments present in MG1655 digestions were no longer visible and the eight fragments resulting from partial cleavage were now represented stoichiometrically. This supports the hypothesis that methylation of certain cytosine residues interferes with cleavage by SfiI.

Figure 3 shows the sequence of the nine $d c m-S f i$ sites in context with surrounding sequence and indicates the observed pattern of cleavage at each site. The $\mathrm{dcm}$ methylation site can overlap either one or two of the internal $\mathrm{C}$ or $\mathrm{G}$ residues (classes I and II, respectively), or it can overlap either one or two of the external C or G residues (classes III and IV, respectively). Each of these four classes is represented at least once in the EcoSeq5 database. The three inefficiently cleaved sites, sS7, sS12 and sS24, all belong to class II. While the sequence of site sS5 is not available, its sensitivity to $\mathrm{dcm}$-mediated methylation would predict that this site is also a class II site. In these cases, both the internal-most cytosine residue in the recognition sequence and the cytosine residue immediately adjacent to the cleavage site are methylated in a $\mathrm{dcm}^{+}$strain. Class IV sites will be methylated on the external-most cytosine residue of the recognition sequence, with the cleavage site unaffected. In the case of class I and class III sites, none of the cytosines in the recognition sequence or adjacent to the cleavage site are methylated. The single class I site and all four class IV sites are cleaved completely in both MG1655 and the $\mathrm{dcm}$ strain. The single representative of class III is not cut by SfiI in either MG1655 or the $\mathrm{dcm}$ strain. Therefore, $\mathrm{dcm}$ methylation is not responsible for the failure of SfiI to cleave this site.

The data from these sites is consistent with the hypothesis that SfiI is sensitive to methylation of the internal-most cytosine residue in the recognition sequence. Alternatively, SfiI may recognize the methylated sequences but be unable to cleave on the $3^{\prime}$ side of a methylated cytosine residue. Because SfiI is otherwise insensitive to the base composition of the cut site, the former explanation seems more likely. This explanation is compatible with the data of McClelland and Nelson, who found that several externally overlapping $\mathrm{dcm}$ methylation sites did not affect the activity of SfiI (25). Finally, partial cleavage at these sites can be explained in one of two ways. One, SfiI may be able to recognize and cleave internally methylated sites, but with a lower efficiency. Two, SfiI may not recognize or cleave internally methylated sites at all and the partial cleavage may represent a small population of these sites that are not methylated.

\section{CONCLUSION}

A complete SfiI restriction map of Escherichia coli K-12 strain MG1655 has been constructed. Pulsed field gel electrophoresis was combined with several other techniques to produce this map. These include analysis of a set of twenty-six auxotrophic transposon insertion mutants, probing of Gene Mapping Membranes, analysis of the EcoSeq5 DNA sequence database, and correlation with the EcoMap5 physical map of $E$. coli $\mathrm{K}-12$. The thirty-one mapped SfiI sites, along with the twenty-two NotI sites previously mapped, bring to fifty-three the number of mapped rare-cutting restriction enzyme sites in this common laboratory strain of $E$. coli that have been placed on EcoMap5. These two maps may prove useful in the physical mapping of point mutations, as well as in the study of larger chromosomal rearrangements, such as inversions, deletions and duplications. As an example, our lab is currently using these maps to analyze duplication formation and resolution in the $E$. coli chromosome. We are also undertaking a comparison of several laboratory strains of $E$. coli using the combined maps. This comparison will address in part the extent to which physical mapping data from MG1655 can be applied to other K-12 strains.

\section{ACKNOWLEDGMENTS}

Our thanks go to K. Rudd and B. Bachmann for communication of unpublished data. Special thanks go to P. Berget, R. Maurer, and the students of the Advanced Bacterial Genetics course taught at Cold Spring Harbor Laboratory for their help in developing and applying many of the transposition and mapping techniques used to isolate and analyze auxotrophs. This work is supported in part by PHS Grant GM35247 to G.M.W.

\section{REFERENCES}

1. Bachmann, B.J. (1990) Microbiol. Rev., 54, 130-197.

2. Kohara, Y., Akiyama, K. and Isono, K. (1987) Cell, 50, 495-508.

3. Kohara, Y. (1990) In Drlica, K. and Riley, M. (ed.), The Bacterial Chromosome. American Society for Microbiology, Washington, D.C., pp. 29-42.

4. Rudd, K.E., Miller, W., Ostell, J. and Benson, D.A. (1990) Nucl. Acids Res., 18, 313-321.

5. Rudd, K.E., Miller, W., Werner, C., Ostell, J., Tolstoshev, C. and Satterfield, S.G. (1991) Nucl. Acids Res., 19, 637-647.

6. Noda, A., Courtright, J.B., Denor, P.F., Webb, G., Kohara, Y. and Ishihama, A. (1991) BioTechniques, 10, 474-477.

7. Hill, C.W. and Harnish, B.W. (1981) Proc. Natl. Acad. Sci. USA, 78, 7069-7072.

8. Bachmann, B.J. (1987) In Neidhardt, F.C., Ingraham, J.L., Low, K.B., Magasanik, B., Schaechter, M. and Umbarger, H.E. (ed.), Escherichia coli and Salmonella typhimurium: Cellular and molecular biology. American Society for Microbiology, Washington, D.C., pp. 1190-1219.

9. Clowes, R.C. and Hayes, W. (1968) Experiments in Microbial Genetics. Blackwell Scientific Publications, Oxford. 
10. Guyer, M.S., Reed, R.R., Steitz, J.A. and Low, K.B. (1981) Cold Spring Harb. Symp. Quant. Biol., 45, 135-140.

11. Singer, M., Baker, T.A., Schnitzler, G., Deischel, S.M., Goel, M., Dove, W., Jaacks, K.J., Grossman, A.D., Erickson, J.W. and Gross, C.A. (1989) Microbiol. Rev., 53, 1-24.

12. Daniels, D.L. and Blattner, F.R. (1987) Nature, 325, 831-832.

13. Daniels, D. (1990) Nucl. Acids Res., 18, 2649-2651.

14. Heath, J.D., Perkins, J., Sharma, B. and Weinstock, G. (1992) J. Bacteriol., 174, 558-567.

15. Chu, G., Vollrath, D. and Davis, R.W. (1986) Science, 234, 1582-1585.

16. Devereux, J., Haeberli, P. and Smithies, O. (1984) Nucl. Acids Res., 12. 387-395.

17. Wu, H.-M. and Crothers, D.M. (1984) Nature, 308, 509-513.

18. Smith, C.L. and Kolodner, R.D. (1988) Genetics, 119, 227-236.

19. Condemine, G. and Smith, C.L. (1990) In Drlica, K. and Riley, M. (ed.), The Bacterial Chromosome. American Society for Microbiology, Washington, D.C., pp. 53-60.

20. Médigue, C., Viari, A., Hénaut, A. and Danchin, A. (1991) Mol. Microbiol., 5, 2629-2640.

21. Carlson, J., Fuchs, J.A. and Messing, J. (1984) Proc. Natl. Acad. Sci. U.S.A., 81, 4294-4297.

22. Skovgaard, O. (1990) Gene, 93, 27-34.

23. Walker, J.E., Gay, N.J., Saraste, M. and Eberle, A.N. (1984) Biochem. J., 224, 799-815.

24. Smith, C.L., Econome, J.G., Schutt, A., Klco, S. and Cantor, C.R. (1987) Science, 236, 1448-1453.

25. McClelland, M. and Nelson, M. (1988) Gene, 74, 291-304. 